\title{
Extended magnetic reconnection across the dayside magnetopause
}

Article

Published Version

Dunlop, M. W., Zhang, Q.-H., Bogdanova, Y. V., Lockwood, M., Pu, Z., Hasegawa, H., Wang, J., Taylor, M. G. G. T., Berchem, J., Lavraud, B., Eastwood, J., Volwerk, M., Shen, C., Shi, J.K., Constantinescu, D., Frey, H., Fazakerley, A., Sibeck, D., Escoubet, P., Wild, J. A. and Liu, Z.-X. (2011) Extended magnetic reconnection across the dayside magnetopause. Physical Review Letters, 107 (2). 025004. ISSN 0031-9007 doi: https://doi.org/10.1103/PhysRevLett.107.025004 Available at https://centaur.reading.ac.uk/22912/

It is advisable to refer to the publisher's version if you intend to cite from the work. See Guidance on citing.

Published version at: http://dx.doi.org/10.1103/PhysRevLett. 107.025004

To link to this article DOI: http://dx.doi.org/10.1103/PhysRevLett.107.025004

Publisher: American Physical Society

All outputs in CentAUR are protected by Intellectual Property Rights law, including copyright law. Copyright and IPR is retained by the creators or other copyright holders. Terms and conditions for use of this material are defined in the End User Agreement. 


\section{CentAUR}

Central Archive at the University of Reading

Reading's research outputs online 


\title{
Extended Magnetic Reconnection across the Dayside Magnetopause
}

\author{
M. W. Dunlop, ${ }^{1,12,14, *}$ Q.-H. Zhang, ${ }^{2}$ Y. V. Bogdanova, ${ }^{3}$ M. Lockwood, ${ }^{1}$ Z. Pu, ${ }^{4}$ H. Hasegawa, ${ }^{5}$ J. Wang, ${ }^{4}$ \\ M. G. G. T. Taylor, ${ }^{6}$ J. Berchem, ${ }^{7}$ B. Lavraud, ${ }^{9}$ J. Eastwood, ${ }^{14}$ M. Volwerk, ${ }^{10}$ C. Shen,${ }^{12}$ J.-K. Shi, ${ }^{12}$ \\ D. Constantinescu, ${ }^{8}$ H. Frey, ${ }_{11}^{1}$ A. N. Fazakerley, ${ }^{3}$ D. Sibeck, ${ }^{13}$ P. Escoubet, ${ }^{6}$ J. A. Wild, ${ }^{15}$ and Z.-X. Liu ${ }^{12}$ \\ ${ }^{1}$ Rutherford-Appleton Laboratory, Chilton, Oxfordshire, OX11 0QX, United Kingdom \\ ${ }^{2}$ SOA Key Laboratory for Polar Science, Polar Research Institute of China, Shanghai, China \\ ${ }^{3}$ Mullard Space Science Laboratory, University College London, Dorking, Surrey, United Kingdom \\ ${ }^{4}$ School of Earth and Space Sciences, Peking University, Beijing, 100871, China \\ ${ }^{5}$ Department of Space Plasma Physics, ISAS/JAXA, Japan \\ ${ }^{6}$ ESA/ESTEC, 2200 AG Noordwijk, The Netherlands \\ ${ }^{7}$ IGPP-UCLA, 3877 Slichter Hall, Los Angeles, California 90095-1567, USA \\ ${ }^{8}$ Institut für Geophysik und Meteorologie, TU-BS, D-38106 Braunschweig, Germany \\ ${ }^{9}$ CESR-CNRS, 9 Avenue du Colonel Roche, 31028 Toulouse Cedex 4, France \\ ${ }^{10}$ Space Research Institute, Austrian Academy of Sciences, Schmiedlstrasse 6, 8042 Graz, Austria \\ ${ }^{11}$ Space Sciences Lab., University of California, 7 Gauss Way, Berkeley, California 94720-7450, USA \\ ${ }^{12}$ CSSAR, CAS, PO Box 8701, Beijing 100190, China \\ ${ }^{13}$ Code 674, NASA/GSFC, Greenbelt, Maryland 20771 USA \\ ${ }^{14}$ The Blackett Laboratory, Imperial College London, London, SW7 2AZ, United Kingdom \\ ${ }^{15}$ Space Plasma Environment and Radio Science group, Lancaster University, LA1 4WA, United Kingdom
}

(Received 29 December 2010; published 6 July 2011)

\begin{abstract}
The extent of where magnetic reconnection (MR), the dominant process responsible for energy and plasma transport into the magnetosphere, operates across Earth's dayside magnetopause has previously been only indirectly shown by observations. We report the first direct evidence of $X$-line structure resulting from the operation of MR at each of two widely separated locations along the tilted, subsolar line of maximum current on Earth's magnetopause, confirming the operation of MR at two or more sites across the extended region where MR is expected to occur. The evidence results from in-situ observations of the associated ion and electron plasma distributions, present within each magnetic $X$-line structure, taken by two spacecraft passing through the active MR regions simultaneously.
\end{abstract}

DOI: 10.1103/PhysRevLett.107.025004

PACS numbers: 94.05. $-\mathrm{a}, 52.20 .-\mathrm{j}, 94.30 . \mathrm{ch}, 94.30 . \mathrm{cq}$

The transport of plasma and energy into Earth's magnetospheric environment, bounded by the magnetopause (MP), is known to be dominantly controlled by the process of magnetic reconnection (MR, [1]) of the Earth's dayside magnetic field with the adjacent magnetosheath magnetic field (the interplanetary field draped over the magnetopause by the impact of the solar wind passing through the upstream bow shock). Previous investigations have raised questions regarding the location and rate of MR across the MP surface [2], particularly in terms of global ionospheric convection as driven by the operation of reconnection. Much of the indirect experimental evidence suggests a patchy or sporadic occurrence, resulting in intermittent reconnection signatures [3], while different theories place the likelihood of MR at a magnetic merging line, which may be tilted along the direction of maximum current on the MP surface, or where these fields are strictly antiparallel [4]. These locations are controlled by the relative orientations of the reconnecting magnetic fields in the magnetosphere and magnetosheath which define a shear angle (which takes different values at different positions on the magnetopause). The biggest differences in the predictions of these theories occurs at the subsolar magnetopause, although there is a large body of indirect evidence, which suggests multiple locations, or extended regions, of MR exist, particularly extending to flank MP positions [5]. In fact, a number of recent studies have implied MR is occurring at both antiparallel and component sites $([6,7])$ and so the nature and extent of reconnection at the subsolar magnetopause remains to be clarified.

The center of the merging current line is typically located near the subsolar region, depending on the draping and orientation of the magnetosheath field, and is controlled by the presence of a guide field during component MR. The operation of MR along this line is expected to result in magnetic $X$-line formation (often localized in extent and with characteristic ion and electron diffusion regions and a central, null field). Associated bundles of reconnected magnetic flux form flux tubes, moving in predictable ways from the merging line during periods of ongoing or intermittent reconnection (e.g., [8]). Until now, direct evidence of the extent of any $X$-line structure has been limited, although theoretical considerations of 3D MR predict an $A-B$ null-null line [9], with characteristic plasma distributions and a system of Hall currents ([10-13]). It is not well understood, however, how current 
theory (applicable to MR sites in the geomagnetic tail) is fully adapted to the driven conditions at the dayside MP [10b], nor any dependence on location. Furthermore, observations of significant numbers of reconnected flux tubes at diverse locations on the MP seem to favor the formation of multiple patches of active MR along the merging line.

The measurements presented here demonstrate the simultaneous existence of $X$-line reconnection structure at widely separated positions $\left(\sim 9 R_{E}\right)$ exactly along the predicted subsolar merging line (either side of noon) for the first time, and hence imply that driven MR activity exists across the low-latitude dayside MP. The evidence arises from a simultaneous MP encounter by two widely separated spacecraft, which each cross the dayside plasma boundary layer close to the diffusion region at each site; one very near to the null field. The in situ, time series measurements are taken from the Double Star (DSP), TC-1 [14] and the THEMIS A spacecraft [15], which were both moving outbound from the Earth's magnetosphere at low latitudes on 14 June 2007 at around 4:40 UT. Each spacecraft carries a complement of plasma and field instrumentation, including fluxgate magnetometers, which provide calibrated data, accurate to below $0.1 \mathrm{nT}([16,17])$. We use temporally averaged data at a time resolution of one vector every 4 seconds for TC- 1 and 3 seconds for THEMIS A. These magnetic field data are shown between 3 and 5 UT in Fig. 1, which also shows the magnetic field from the other four THEMIS spacecraft. In fact, these measurements are part of a wider sampling of the MP achieved by 10 spacecraft (five THEMIS, TC-1, and four Cluster, [6b])

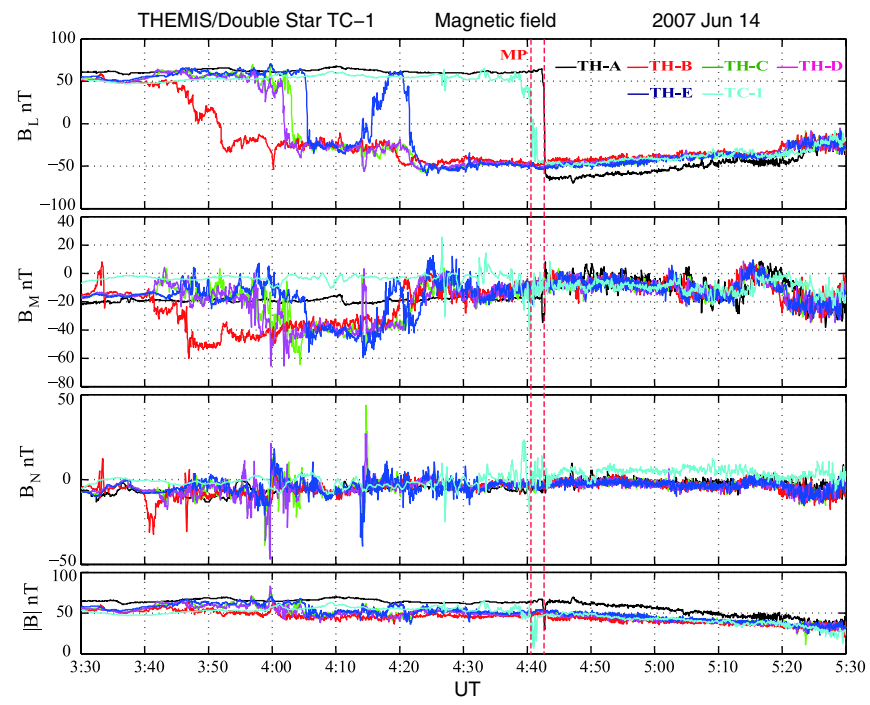

FIG. 1 (color online). Magnetic field data, taken during the time interval around the MP crossing of the spacecraft in magnetopause aligned "LMN" coordinates, where $N$ is the magnetopause normal, $L$ is in the $\left(Z_{\mathrm{GSM}}, N\right)$ plane and $M$ completes a left-handed set (GSM is the geocentric solar magnetospheric coordinate system). The reversal in the $B_{L}$ components marks the crossing (vertical red line). Also shown are data from the other four THEMIS spacecraft $(B, C, D$ and $E$ ), which cross the MP just before THEMIS A in sequence. distributed around the MP to the dawnside flank, which suggest MR is operating quasisimultaneously at both these central, dayside locations, as well as at low-latitude, (originally antiparallel) flank locations.

Figure 1 shows sharp reversals in the $B_{L}$ components (aligned to the MP surface) at the times of each spacecraft crossing, marking the exits from the magnetosphere to the magnetosheath. The actual crossing velocity corresponds mainly to an inward motion of the MP, resulting from an increase in the solar wind dynamic pressure during this time. We place the exits of THEMIS A and TC- 1 just north of any reconnection site, since both appear to see northward moving reconnected flux tubes (inferred from $+/-$ polarity, bipolar signatures in the $B_{N}$ component, see, e.g., [8], which are small scale on THEMIS A), suggesting timedependent, low-latitude MR is occurring just south of both locations [but see also later discussion of northward ion jets shown in Fig. 3(a)]. The other THEMIS spacecraft precede THEMIS A, exiting into the magnetosheath one by one. Although the data show rather more complicated signatures at the earlier time of these crossings, they also show strong bipolar signatures (at 4:00 and 4:12 UT [18]), but with $-/+$ polarity, characteristic of the southward passage of reconnected flux tubes generated by time-dependent MR.

Both spacecraft, TC-1 and TH-A, therefore cross the magnetopause within 2 minutes of each other. In fact, the interplanetary magnetic field (IMF), which ultimately controls the magnetosheath field orientation, rotates from a strong $B_{Y}$ (Eastward pointing) to a strong- $B_{z}$ (Southward pointing) orientation, in the hour before the crossing. This southward orientation suggests that the low-latitude merging line would reform to take an orientation close to EastWest, as modeled in Fig. 2(a), which also shows the positions of each spacecraft at the MP, as seen from the sun. The model shown, predicts the subsolar location of maximum current arising from the crossed magnetospheric and draped magnetosheath fields either side of the MP boundary, fitted for the particular conditions at the time of the crossings. The actual merging line may be slightly curved, rather than as simply drawn here. The expected directions of motion of any reconnected flux tubes moving away from this line are then computed. Actual flux tube motions from the observed bipolar signatures, where available, were checked by de Hoffmann-Teller analysis at each spacecraft and are consistent with the model directions shown. The merging line therefore threads through each position, as shown in Fig. 2(a).

The observational evidence presented below strongly suggests that each spacecraft passes very close to a possible $X$-line structure, as indicated by the schematic in Fig. 2(b) and each of which are ordered to the merging line at each site (drawn, for convenience, as one structure in the diagram). The extent and connection of these local structures along the merging line of maximum current is not known, since single or multiple reconnection lines could exist. In this scheme, however, both spacecraft pass from an initially closed field region inside the 

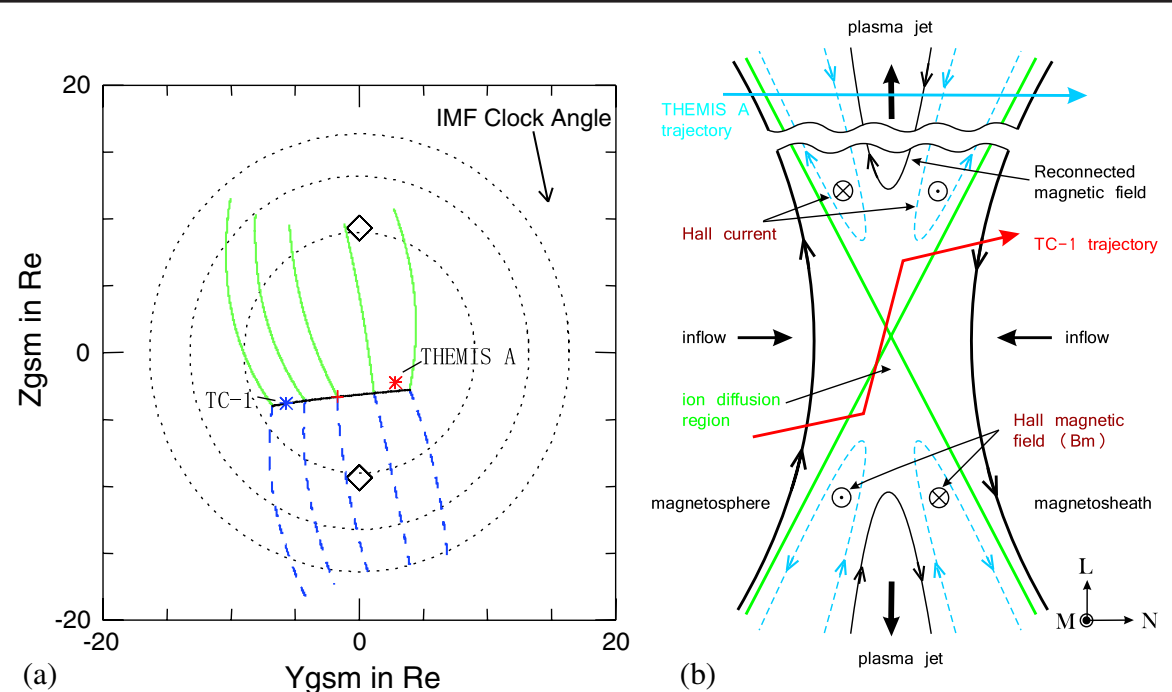

FIG. 2 (color online). (a) Conditions at the magnetopause during the simultaneous MP encounter connected by the same merging current line at locations separated by $\sim 9 R_{E}$. The TC-1 spacecraft is marked by the blue star; the Th-A spacecraft by the red star, and the view is of the Earth's magnetopause from the Sun, so that the projection is in the $(Z, Y)$ plane (where $X$ points from the Earth to the Sun). The diamonds mark the position of the magnetic polar cusps and the curved tracks are the predicted motions of reconnected flux tubes arising from positions along the merging line, which is fitted to the ambient, draped magnetosheath field [19]. The motions depend on a model for the field line tension and magnetosheath flow. (b) Schematic of the inferred (simplified) tracks of each spacecraft relative to their local reconnection $X$-line structure (after 10). The diagram is broken to indicate that the precise position of Th-A is not known. Note that the spacecraft pass near each $X$-line at different locations along the predicted merging current line (out of the plane of the paper).
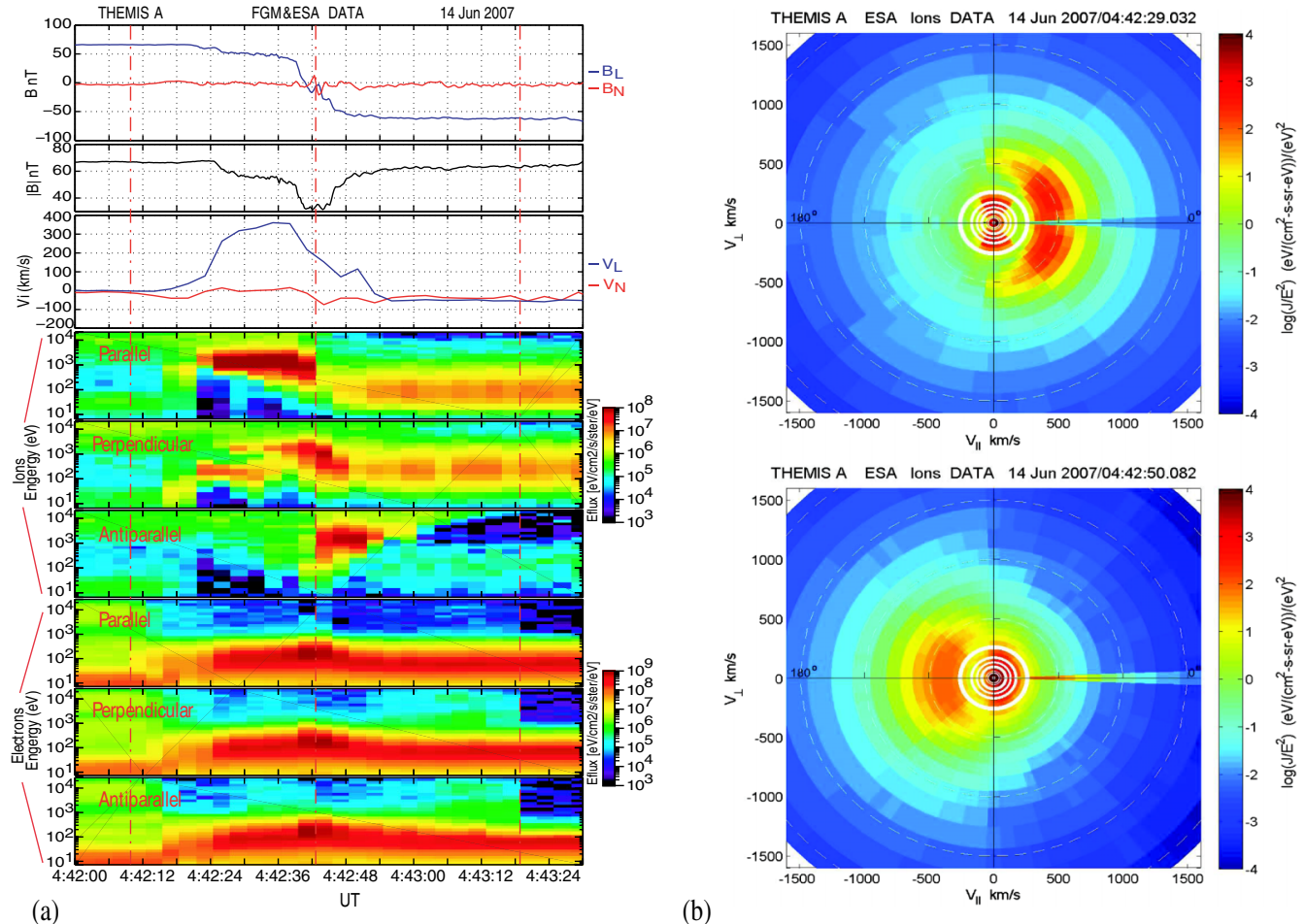

FIG. 3 (color online). (a) THEMIS-A energy spectra for both ions and electrons, separated into angular ranges surrounding the directions parallel, perpendicular and antiparallel to the magnetic field. The six lower panels on the left show these data for the time interval surrounding the boundary crossing, with the magnetic field magnitude, $B_{N}$ and $B_{L}$ components, and $V_{L}$ and $V_{N}$, the bulk ion flows, shown in the top three panels. The three vertical dashed lines indicate the inner and outer boundaries of the reconnection layer and the location of the current sheet (middle line). (b) Shows the 2-D ion velocity, perpendicular and parallel to the magnetic field, for times either side of the current sheet. These distributions are reflected about $V_{\perp}=0$. 


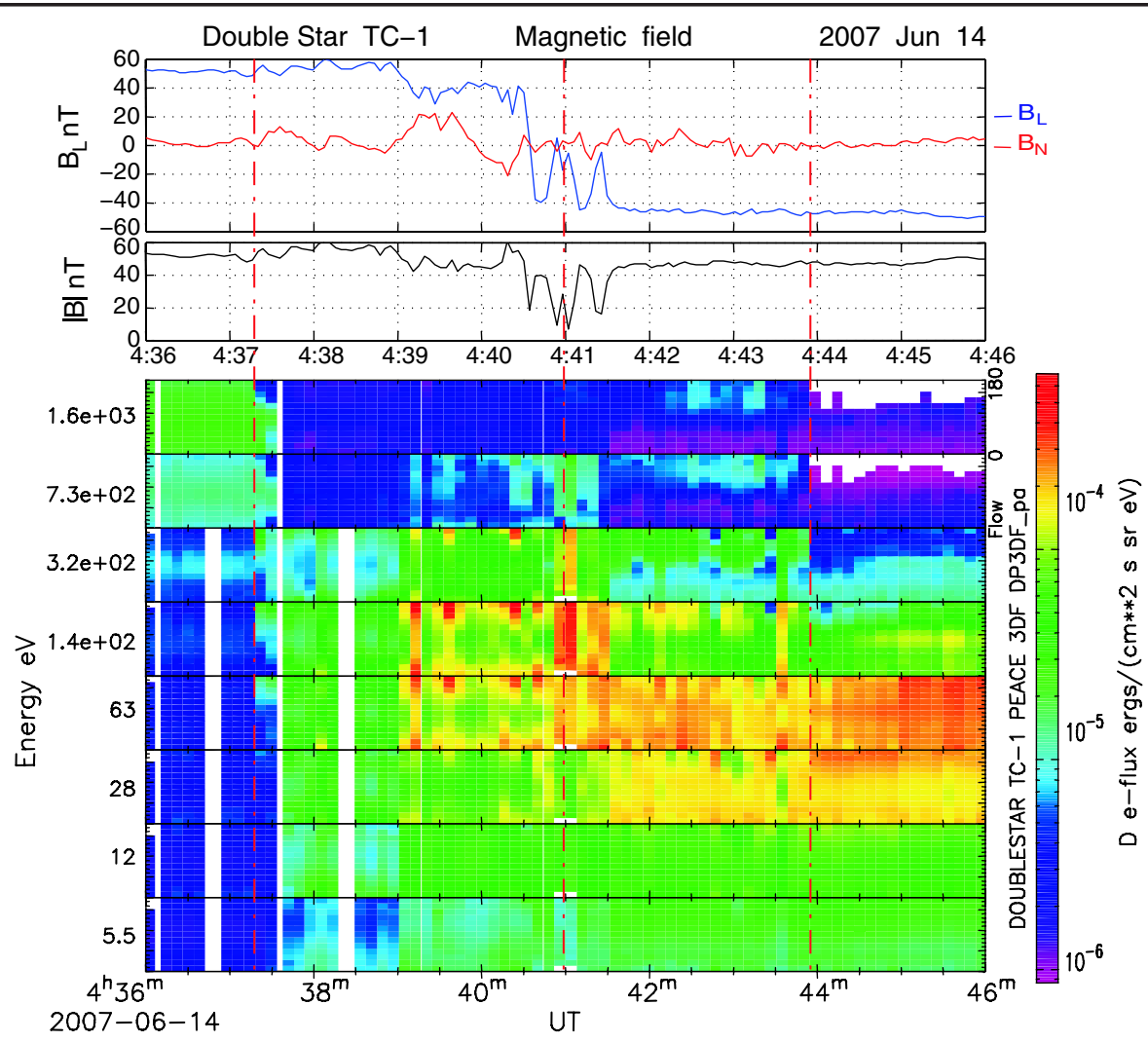

FIG. 4 (color online). The differential energy flux of the TC-1 electron distribution is shown on the bottom panels. Each subpanel shows the pitch angle spectrogram for an energy range indicated on the left. The pitch angle is the angle between the vector of the particle velocity and the magnetic field vector. Each subpanel, therefore shows the populations of electrons flowing at each angle to the field (0-180 deg, from bottom to top of each subpanel). The top panels show the magnetic field magnitude, $B_{N}$ and $B_{L}$ components.

magnetosphere, sampling in turn the most recently reconnected field on the magnetospheric side of the current sheet, the ion outflow region and then the most recently reconnected field again (this time on the magnetosheath side). The expected system of Hall currents may be significantly modified from this schematic in the case of the magnetopause [10b], or in the presence of a strong guide field [20], as is the case here, although the determination of Hall signatures is not clear from the observations here. The evidence suggests that THEMIS A, at its location, passes further from the null field than TC-1, which appears to pass initially south of the $X$-line, on the inner edge of the separatrix; moving north before its exit, as indicated schematically in the figure, with the implication that TC-1 passes very close to, or within the diffusion region, rather than across the ion outflow.

The presence of a reconnection structure can be probed using thermal plasma measurements taken by the ion and electron spectrometers onboard each spacecraft $([21,22])$. These instruments measure the full 3D distribution of the thermal plasma populations in the energy range $4 \mathrm{eV}$ $25 \mathrm{keV}$. For THEMIS A, Fig. 3 shows plasma signatures, consistent with $X$-line formation close by. At times earlier than the first boundary ( $4: 42: 10$ UT), the data shows ion and electron, magnetospheric populations (above $1 \mathrm{keV}$ ), which change as the spacecraft cross into the reconnection layer. At times later than the right hand boundary (4:43:19 UT), low temperature, incoming magnetosheath plasma is observed, which is increasingly modified at earlier times as the spacecraft crosses reconnected field lines. The minimum in the magnetic field magnitude, and reversal in $B_{L}$, marks the central current sheet (4:42:43 UT) and $V_{L}$ shows clear ion outflow, peaking just inside this boundary. At this time there is a small-scale, bipolar $B_{N}$ signature, suggesting a northward moving flux rope. There is distinct plasma ion structure either side of this boundary: in the boundary layer, all particle populations depend on the distance from the center of the current sheet, and hence on time elapsed since reconnection, as predicted by theory ([23,24]). For the electrons, the main population is clearly energized, peaking at the current sheet, but there are also characteristic anisotropic populations present, which show clear observations of the escaping magnetospheric electrons on recently reconnected field lines (inside the outer boundary of the reconnected layer [25]).

In the ion outflow region surrounding the current sheet (about 4:42:20-4:43:15 UT), the predominant ion populations show strong acceleration along the magnetic field which is consistent with the ion " $D$ " distribution predicted by [26], as a result of reflection and transmission through the current layer. This acceleration produces an ion population on the inner side of the reconnection layer 
(before 4:42:43 UT), extending through the parallel and perpendicular directions, which is energised to 4 times the incoming magnetosheath energy (twice the magnetosheath bulk velocity); the reflected ion population (energized back-streaming ions) on the outer side of the reconnection layer (after 4:42:43 UT) appearing in the antiparallel direction. The energy range of both these populations shows a low energy cutoff which is also consistent with time of flight from the reconnection site ([23,24]). The $D$ form of these accelerated populations may be seen in the plots on the right-hand side of Fig. 3. The top plot, in particular, shows the characteristic $D$ shape of accelerated ion population extending from the parallel to the perpendicular directions, while the lower plot shows the distribution for the reflected ion population.

Figure 4 shows the boundary layer seen by TC-1, $\sim 9 R_{E}$ along the expected merging line from THEMIS A. The ion data from TC-1 was not taken for this interval; however, the electron distribution shown indicates the presence of a reconnection layer and that the spacecraft passed through the $X$ line very near the null field point (as indicated by the dropout of the field magnitude and peak in electron energization) and possibly inside the diffusion region, consistent with the schematic in Fig. 2(b). The electron distribution shows a clear boundary at 4:37:20 UT, where the spacecraft move from high energy, magnetospheric electrons, into a region of anisotropic distributions, which show both bistreaming and beamed populations, particularly after 4:39 UT. The initial population, before 4:39 UT, also contains field antiparallel electron flows at accelerated magnetosheath energies. These electrons should be flowing inwards towards the ionosphere, so that the field directions in the scheme in Fig. 2(b) place TC-1 just south of the $X$-line, initially (afterwards TC- 1 is north (the $X$-line moves south) and antiparallel distributions are outflowing). This first boundary is interpreted as before as the first entry into the reconnection layer (field lines opened by reconnection). We place the current sheet at 4:41 UT, where $B_{L}$ reverses and the field minimum occurs, and the outer edge of the electron boundary layer at 4:44 UT, where the magnetosheath population is recovered. Either side of the current sheet, the distribution shows much structure, but there is a consistent out-flowing population in the region after the current sheet, suggesting the presence of reflected magnetosheath electrons (between 28-320 eV) and outflowing magnetospheric electrons (above $730 \mathrm{eV}$ ) on field lines which have been opened by MR. Such acceleration and reflection of electrons in the mid energy range are often observed on recently reconnected field lines [25].

In the region before the current sheet, the bidirectional and beamed character, alternates between field parallel and antiparallel directions, at different times and in different energy bands, and corresponds in time to the bipolar signature in $B_{N}$. Although the $X$-line could be moving north to south across TC- 1 during this time, this behavior is not inconsistent with the existence of quadrupolar Hall currents (which have been recently demonstrated statistically in situ [20]). However, it is not so significant on the outer side of the boundary (when the $X$-line could be moving southward). The energy profiles of the electron distributions are similar for both TC- 1 and THEMIS A, although the TC-1 profile is consistent with its path crossing close to, or within, the central diffusion region (note the high acceleration at the drop out of the field magnitude). The temporal scale of the boundary layer is clearly different at each site, and there is some evidence, from computed magnetopause crossing speeds, to suggest that the boundary motion at THEMIS is high. Nevertheless, although this suggests the boundary layer is thicker at TC-1 (possibly caused by a higher reconnection rate), it is consistent with the angled path of TC-1 from south to north [as indicated on Fig. 2(b)], which requires the spacecraft to remain in the reconnection layer longer. Single spacecraft estimates of spatial scale are therefore prone to errors. These relative motions and in-situ parameters, however, suggest the ion inertial scale is $\sim 70 \mathrm{~km}$, while the overall reconnection layer is $\sim 0.5 R_{E}(\sim 50 x$ the ion scale $)$ and place the central current sheet at a few ion inertial scale lengths.

Analysis of in-situ magnetic field and plasma data has therefore confirmed the simultaneous operation of MR at two widely separated sites along the expected, low-latitude merging line stretching across the day side, subsolar region of the magnetopause. These two reconnection sites may not be connected if only patchy reconnection is active along the merging line. Both electron and ion energisation and reflection are observed within each reconnection structure, together with characteristic ion-D distributions, which confirm $X$-line formation with significant extent. These data are therefore consistent with the presence of multiple (two or more) patches of MR along the expected merging line, since a single $X$-line over a distance of more than $9 R_{E}$ is unlikely. The purpose here has therefore been primarily to confirm the extended operation of MR along the low-latitude merging line, as the most probable location of MR activity. In fact, the activity occurs under conditions when MR sites appeared to be also present at flank locations, earlier. This is the first time these mechanisms have been directly shown (in situ) to operate at multiple or extended locations across the dayside region.

M. Dunlop is partly supported by the Chinese Academy of Sciences through grant no. 2009S1-54) and through a working group sponsored by ISSI, Berne. Q. H. Zhang was supported by the STFC in the UK and by the CSOA in China (No. 201005017). J. P.E. received support from the STFC (Grant No. ST/G00725X/1) at ICL. Z. Pu and J. Wang are supported by the CNSF grants 40731056, 40974095.

*m.w.dunlop@rl.ac.uk

[1] J. W. Dungey, Phys. Rev. Lett. 6, 47 (1961).

[2] R. L. Kessel et al., Geophys. Res. Lett. 23, 583 (1996). 
[3] G. Paschmann et al., Nature (London) 282, 243 (1979).

[4] K. J. Trattner et al., J. Geophys. Res. 112, A08210 (2007).

[5] T-D. Phan et al., Nature (London) 404, 848 (2000); Geophys. Res. Lett. 33, L09104 (2006).

[6] M. W. Dunlop et al., Phys. Rev. Lett. 102, 75005 (2009); Ann. Geophys. (to be published).

[7] Z.-Y. Pu et al., Geophys. Res. Lett. 34, L20101 (2007).

[8] M. W. Dunlop et al., Ann. Geophys. 23, 2867 (2005).

[9] C. J. Xiao et al., Nature Phys. 3, 609 (2007).

[10] F. S. Mozer et al., Phys. Rev. Lett. 89, 015002 (2002); Geophys. Res. Lett. 35, L17S04 (2008).

[11] T-D. Phan et al., Geophys. Res. Lett. 34, L14104 (2007).

[12] Oiereset et al., Nature (London) 412, 414 (2001).

[13] Vaivads et al., Phys. Rev. Lett. 93, 105001 (2004).

[14] Z.X. Liu et al., Ann. Geophys. 23, 2707 (2005).

[15] V. Angelopoulos, Space Sci. Rev. 141, 5 (2008).
[16] A. U. Auster et al., Space Sci. Rev. 141, 235 (2008).

[17] C. Carr et al., Ann. Geophys. 23, 2713 (2005).

[18] H. Hasegawa et al., Geophys. Res. Lett. 37, L16101 (2010).

[19] B. Cooling et al., J. Geophys. Res. 106, 18763 (2001).

[20] Eastwood et al., Phys. Rev. Lett. 104, 205001 (2010); J. Geophys. Res. 115, A08215 (2010).

[21] J. P. McFadden et al., Space Sci. Rev. 141, 277 (2008).

[22] A. N. Fazakerley et al., Ann. Geophys. 23, 2733 (2005).

[23] M. Lockwood and M. F. Smith, J. Geophys. Res. 99, 8531 (1994).

[24] M. Lockwood and M. A. Hapgood, J. Geophys. Res. 103, 26453 (1998).

[25] B. Lavraud et al., J. Geophys. Res. 111, A05211 (2006).

[26] S. W. H. Cowley et al., Rev. Geophys. Space Phys. 20, 531 (1982). 